\title{
Retrospective application of prognostic indices to pancreatitis discovered at necropsy
}

\author{
M K Heatley
}

\begin{abstract}
Six prognostic indices, which were developed to assess inpatients with acute pancreatitis, were evaluated for possible retrospective application. When applied to a series of 14 cases in whom pancreatitis was first diagnosed at necropsy, the index devised by Jacobs et al was found to be the most useful, because in nine of these cases eight or more of the variables required were available for assessment from the case records. In the other indices evaluated fewer than eight of the required variables were available for retrospective assessment in most cases.
\end{abstract}

Although undiagnosed pancreatitis is probably uncommon as a sole cause of death, the retrospective use of one or more of these indices may help assess the severity of the patient's condition on admission to hospital.

Acute pancreatitis is often first diagnosed at necropsy.' It might not be diagnosed in life because the patient presents with atypical clinical features, or because the predominant symptoms are those of a complication of pancreatitis, such as respiratory failure. The pathologist may then be asked if, as a result of the failure to make the diagnosis in life, the patient died because he or she did not receive appropriate treatment.

Severe acute pancreatitis is fatal in a high proportion of cases, even where appropriate medical treatment is given. ${ }^{2}$ Mild acute pancreatitis is much less serious, although inappropriate treatment such as the reintroduction of oral feeds can result in the disease being reactivated and evolving into a more serious and potentially fatal form of pancreatitis. ${ }^{3}$ The essentially similar pathological findings in both types of patients are likely to be unhelpful in discriminating between the two groups as they will both simply show end stage pancreatitis with extensive necrosis of Accepted for publication 18 July 1990

Table 1 Number of cases out of 14 in which related numbers of variables were available

\begin{tabular}{llllllllll}
\hline Number of variables & 1 & 2 & 3 & 4 & 5 & 6 & 7 & 8 & 9 \\
\hline Agarwal (7) & 0 & 1 & 2 & 6 & 3 & 2 & 0 & - & - \\
Cassey (9) & 0 & 1 & 0 & 2 & 1 & 2 & 2 & 1 & 5 \\
Imrie (8) (modified) & 1 & 2 & 2 & 1 & 5 & 1 & 2 & 0 & 4 \\
Jacobs (11) & 0 & 0 & 1 & 0 & 2 & 1 & 1 & 5 & 2 \\
Ranson (11) & 0 & 2 & 0 & 0 & 2 & 2 & 3 & 3 & 5 \\
Schuppiser (11) & 0 & 0 & 2 & 1 & 1 & 1 & 3 & 1 & \\
\hline
\end{tabular}

Figures in parentheses are numbers of variables required for a complete assessment by this method. the parenchyma, associated with an intense inflammatory infiltrate.

The question, however, might be elucidated by the retrospective application of one of several indices developed for assessing the clinical condition of newly admitted patients diagnosed in life as having acute pancreatitis. The use of these clinical indices at necropsy in previously undiagnosed cases of pancreatitis may present difficulties on two counts. Firstly, they may require the results of complex laboratory investigations which are not routinely carried out on admission to hospital. Secondly, some of these indices require the use of serial measurements over 24 or 48 hours. These may not be available, particularly if the patient died shortly after admission.

An ideal index for the retrospective assessment of the clinical condition of the patient with unsuspected pancreatitis would therefore be designed to rely on commonly measured physiological, haematological, and biochemical variables which would usually be assessed and recorded in the case sheet in any newly admitted patient, irrespective of diagnosis.

To identify the index which would be most suitable for this purpose, six established clinical indices were applied retrospectively to each of 14 patients who had died between 1971 and 1984 with an acute pancreatitis first diagnosed at necropsy. A complete clinical record was available for each patient from the time of admission to death. The number of the required variables actually available in the case notes of each patient was counted. ${ }^{24-8}$ For each index, the number of cases in which all of the necessary variables were available, the number in which all but one, and so on, were counted. The results of this assessment are presented in table 1 .

At best, in any of the indices studied, no more than nine of the required variables could be assessed. In the case of Jacobs' index, ${ }^{7}$ eight or nine of the variables were documented in nine of the 14 cases. In one case only three variables could be documented from the available data.

Cassey's index requires measurements of a maximum of nine variables, ${ }^{5}$ which was achieved in five cases, although compared with Jacobs' index, a much less comprehensive assessment was available in the remaining nine patients reviewed.

The results suggest that, overall, Jacobs' index (table 2) allows for a more comprehensive retrospective assessment of the clinical 
Table 2 Physiological, haematological, and biochemical variables used in assessing prognosis in acute pancreatitis

\begin{tabular}{lll}
\hline Systolic hypotension & $<$ & $90 \mathrm{~mm} \mathrm{Hg}$ \\
Tachycardia & $<$ & 140 \\
Fever & $>$ & $38.4^{\circ} \mathrm{C}$ \\
Prothrombin time & $>$ & 14 seconds \\
White cell count & $<$ & $20 \times 10^{9} / 1$ \\
Haematocrit & $<$ & $30 \%$ \\
Serum albumin & $30 \mathrm{~g} / 1$ \\
Serum creatinine & $>$ & $0 \cdot 18 \mathrm{mmol} / 1$ \\
Serum urea & $>$ & $5 \mathrm{mmol} / 1$ \\
Serum bilirubin & $>$ & $69 \mathrm{mmol} / 1$ \\
Serum calcium & $<$ & $2 \mathrm{mmol} / 1$ \\
\hline
\end{tabular}

findings than the other indices studied, although Cassey's index is more likely to be completed. As has been stated above, indices requiring serial assays, or assays such as the $\mathrm{PaO}_{2}$ in arterial blood which are not routinely carried out on admission, were less likely to permit a complete assessment of the patient's condition.

Although undiagnosed pancreatitis is probably uncommon as a sole cause of death, the retrospective use of one or more of these indices may help assess the severity of the patient's condition on admission to hospital and in determining the contribution made towards a fatal outcome by the failure to diagnose and treat the acute pancreatitis.

1 Wilson C, Imrie CW. Deaths from acute pancreatitis: Why do we miss the diagnosis so frequently? Int J Pancreatol 1988;3:273-82.

2 Ranson JHC, Rifkend KM, Roses DF, Fink SD, Eng K, Spencer FC. Prognostic signs and role of operative management in acute pancreatitis. Surg Gynecol Obstet 1974;139:69-81.

3 Ranson JHC. Acute pancreatitis: Pathogenesis, outcome treatment. Clin Gastroenterol 1984;13:843-63.

4 Agarwal N, Pitchumoni CS. Simplified prognostic criteria in acute pancreatitis. Pancreas 1986;1:69-73.

5 Cassey JG, Clark DA. Predictors of severity of attacks of acute pancreatitis. Aust NZ J Surg 1986;56:887-9.

6 Osborne DH, Imrie CW, Carter DC. Biliary surgery in the same admission for gall stone associated acute pancreatitis. Br J Surg 1981;68:758-61.

7 Jacobs ML, Daggett WM, Civetta JM, et al. Acute pancreatitis: Analysis of factors influencing survival. Ann Surg 1977;185:43-51.

8 Schuppiser JP, Grotzinger V, Van der Linden J. Helv Chir Acta 1984;51:661-3.

\title{
HLA antigens in Hungarian patients with idiopathic haemochromatosis
}

\author{
E Czìnk, E K Gyódi, K Német, S Hollán
}

National Institute of Haematology and Blood Transfusion, Daroczi Unit 24, 1113 Budapest, Hungary E Czink

E K Gyódi

K Német

$S$ Hollán

Correspondence to: Dr E Czink.

Accepted for publication 16 August 1990

\begin{abstract}
Thirteen unrelated patients with idiopathic haemochromatosis (eight men, five women) were studied. The diagnosis was based on clinical, biological, and histochemical findings. HLA typing was performed in all 13 and in all of their available first degree relatives $(n=31)$. HLA A3 was present in nine of 13 probands $(69.2 \%$ compared with $18.8 \%$ in the group of 53 healthy blood donors and $22.4 \%$ in a selected Hungarian population $(\mathbf{n}=1910)$. HLA $B 7$ was present in five of 13 probands (38.4\% compared with $11 \cdot 3 \%$ and $14 \cdot 6 \%$ ). An A3B7 antigen association was found in five of 13 patients. The A3B7 haplotype was found in three, A2B12 and A2B38 haplotypes were found twice in 10 genotyped probands. Pedigree studies showed that there was one unafiected homozygote, 24 heterozygotes, and six non-carriers.

Extended family and population studies are necessary to establish the prevalence of the gene in Hungary and an association with haplotypes other than A3B7.
\end{abstract}

Idiopathic haemochromatosis is a recessively transmitted hereditary disease that is characterised by generalised parenchymal iron overload, leading to liver cirrhosis, diabetes mellitus, cardiomyopathy, endocrine dysfunction, arthropathy and skin pigmentation. ${ }^{1}$ The idiopathic haemochromatosis gene is closely linked to the HLA-A locus on chromosome $6^{2}$; A3, B7, and B14 are most strongly associated with it. ${ }^{3}$ The HLA marker for the idiopathic haemochromatosis allele is, in fact, haplotypic. ${ }^{3}$ In a family where one member has idiopathic haemochromatosis the HLA identical sibling should also be affected. ${ }^{4}$

We collected data on HLA distribution in patients with idiopathic haemochromatosis in Hungary so that we could assess the number of affected family members in a given population.

\section{Methods}

Thirteen unrelated patients (eight men and five women) aged 35 to 65 years were studied. Idiopathic haemochromatosis was diagnosed on clinical, biochemical, and histochemical grounds. HLA typing was performed in 13 probands and in all of their available first degree relatives $(n=31)$ through pedigree studies. Relatives sharing both, one, or no HLA haplotypes with the proband were regarded as either homozygous, heterozygous for the idiopathic haemochromatosis gene, or normal (non-carriers). ${ }^{4}$ Determinations of serum iron, iron binding capacity, and serum 